\title{
Patrones espaciales asociados a la infestación comunitaria por vectores de la enfermedad de Chagas
}

\author{
Spatial patterns of human settlement infestation \\ by Chagas disease vectors
}

\author{
Gabriela Ramírez-Hernández* \\ Jean François Mas** \\ Janine M. Ramsey***
}

Recibido el 18 de agosto de 2019; aceptado 4 de noviembre de 2019

\section{Resumen}

Factores socioculturales y ambientales afectan el contacto en el paisaje entre triatominos infectados con Trypanosoma cruzi y la población humana, etiología de la enfermedad de Chagas. En este trabajo se analizan los efectos de la estructura del paisaje sobre la presencia de triatominos (vectores) en asentamientos humanos de una zona rural de Oaxaca, México. Mediante la búsqueda y colecta participativa de vectores, se calcularon índices de infestación en 35 localidades, en 2005 y en 2015. Se elaboró cartografía de cubierta y uso de suelo para ambas fechas y se estimó la cobertura de cada cubierta dentro de diez áreas de influencia. Se encontraron dos especies principales en el ambiente doméstico: Triatoma dimidiata Haplogrupo 2 (Hg2) y Triatoma mazzottii. La altitud, la densidad de vivienda y el área de bosque tropical son factores que permitieron explicar la presencia de cualquier de triatominos en ambiente doméstico. Se identificaron zonas prioritarias para implementar acciones a

* Universidad Nacional Autónoma de México (UNAM), México, correo electrónico: gabriela.ramrezhdz@gmail.com. ORCID: https://orcid.org/0000-0001-7082-8311

** Universidad Nacional Autónoma de México (UNAM), México, correo electrónico: jfmas@ciga.unam.mx. ORCID: https://orcid.org/0000-0002-6138-9879

*** Instituto Nacional de Salud Pública, México, correo electrónico: jramsey@insp.mx. ORCID: https://orcid.org/0000-0002-5950-0830 
corto y mediano plazo y así atender casos actuales y detener la incidencia de esta zoonosis.

Palabras clave: vectores, infestación, paisaje, Trypanosoma cruzi, enfermedad de Chagas.

\begin{abstract}
Environmental and sociocultural factors affect landscape contact between the human population and triatomines infected with Trypanosoma cruzi, etiology of Chagas disease. This paper analyzes the effects of landscape structure on the presence of triatomines (vectors) in human settlements in a rural area of Oaxaca, Mexico. Through participatory search and collection of vectors, infestation rates were calculated in 35 communities, in 2005 and 2015. Cover and land use mapping were developed for both periods and the coverage of each type within ten areas of influence was estimated. Two primary species were found in the domestic environment: Triatoma dimidiata Haplogroup 2 (Hg2) and Triatoma mazzottii. The altitude, density of housing and surface area of tropical forest are factors that explained presence of either triatomine in houses. Priority surveillance areas were identified for short and mediumterm prevention and healthcare attention to eliminate incidence of this disease.
\end{abstract}

Key words: vectors, infestation, landscape, Trypanosoma cruzi, Chagas disease.

\title{
Introducción
}

Presente de forma endémica en 21 países de Latinoamérica, la enfermedad de Chagas ocasiona la muerte de 10600 personas anualmente. Tan solo en México se estima que el número de personas afectadas por la enfermedad es entre uno y dos millones (Sánchez-González et al., 2016). En años recientes la enfermedad ha adquirido gran importancia de carácter urbano y global debido a los movimientos migratorios de las personas que la padecen (Schmunis y Yadon, 2010; WHO, 2015, 2017).

La enfermedad de Chagas es causada por la infección del parásito Trypanosoma cruzi en órganos internos de mamíferos, entre ellos los humanos. Se le conoce como una enfermedad silenciosa ya que desde que se contamina una persona hasta que se desarrollan síntomas o afectación patológica puede pasar entre 5 y 20 años. Durante la fase crónica, una proporción de las personas desarrollarán alteraciones en el tracto intestinal, trastornos neurológicos y cardiacos (Rassi et al., 2010). El principal mecanismo de transmisión del parásito hacia los humanos es el vectorial, es decir, mediante el contacto con las heces de insectos de la subfamilia Triatominae mejor conocidos como chinches o "pic" en México (Ramsey et al., 2015).

El método más efectivo para impedir la incidencia de la enfermedad es la prevención de la transmisión vectorial (WHO, 2017). Las poblaciones del vector están presentes en diferentes tipos de ambientes, sin embargo, el grado en que están en 
contacto con los humanos difiere dependiendo de múltiples variables socioeconómicas, ambientales y culturales (Valdez-Tah et al., 2015a). Se ha documentado que la calidad de la vivienda, las características del área peri-doméstica, la disponibilidad de hospederos domésticos (Ibarra-Cerdeña et al., 2017) y la ubicación de la vivienda son factores que favorecen significativamente la domiciliación de los vectores, es decir, la infestación doméstica o presencia de vectores dentro y en los alrededores de las viviendas (Cohen et al., 2006; Dumonteil et al., 2013; Bustamante et al., 2014; Rossi et al., 2015; López-Cancino et al., 2015). Estas condiciones permiten a los vectores encontrar refugios, sitios idóneos para la reproducción y acceso a recursos alimenticios constantes. Sin embargo, ha sido poco estudiada la influencia de la variación espacio-temporal del paisaje en el proceso de infestación. De acuerdo con Valdez-Tah et al. (2015a), el grado de modificación del paisaje derivado de las diferentes modalidades de su aprovechamiento da como resultado variaciones en las tasas de infestación doméstica. Los procesos de cambio de uso de suelo como la deforestación para la cría extensiva de ganado y la perturbación de la cubierta forestal para la producción de carbón vegetal o extracción de leña están asociados a elevadas tasas de infestación (Vázquez-Prokopec et al., 2012).

La exposición de la población a los vectores constituye el principal factor de peligro para la transmisión del parásito, lo cual sumado a la vulnerabilidad humana, da como resultado un incremento en el riesgo de contacto vector-humano e incidencia de la enfermedad. Considerando que la identificación de los factores que incrementan la probabilidad de infestación doméstica permite el diseño de estrategias de control de las poblaciones de vectores a diferentes escalas espaciales (Vázquez-Prokopec et al., 2012), se planteó la siguiente investigación con la finalidad de comprender cuáles elementos del paisaje, así como sus cambios, están relacionados con la presencia del vector en viviendas de comunidades rurales de Oaxaca. Bajo el marco conceptual de la ecología del paisaje (Castro et al., 2019) se caracterizó espacio-temporalmente la estructura del paisaje para identificar variables de mayor relevancia para la infestación de asentamientos humanos.

\section{Métodos}

\section{Área de estudio}

El municipio de Santos Reyes Nopala, es uno de los 570 que conforman el estado de Oaxaca, México, como se ilustra en la Figura 1. El territorio municipal abarca una extensión de $226 \mathrm{~km}^{2}$ y comprende dos subprovincias fisiográficas — costa del sur y cordillera costera del sur-, en las cuales se distinguen cuatro tipos de vegetación: selva baja caducifolia, selva mediana subcaducifolia, bosque mixto de pino-encino y bosque de encino, en donde las selvas o bosques tropicales tienen mayor representa- 
tividad (INEGI, 2009). El sistema agrícola de tumba-roza y quema, así como las actividades intensivas de recolección de leña han favorecido la predominancia del uso del suelo con fines ganaderos, agrícolas y para el desarrollo de infraestructura. Prevalece la agricultura de temporal como actividad económica en el municipio, seguida del comercio. Los principales cultivos son: maíz, caña, frijol y café (Pérez, 2010).

El elevado grado de marginación que presenta el municipio deriva de importantes rezagos sociales, lo que se traduce en carencia de recursos básicos como agua entubada o red de drenaje, rezago educativo y escasez en los servicios de salud (CONAPO, 2010). De acuerdo con cifras oficiales para los periodos de evaluación, dos tercios de la población total - 10441 habitantes - vive en 35 localidades rurales reportadas para el municipio (INEGI, 2010). Las viviendas en dichas localidades se caracterizan por estar construidas con tablones de madera como paredes, con techo de lámina y piso de tierra. Usualmente, la vivienda es compartida con animales domésticos como gallinas y mascotas (perros y gatos), y dentro se suele almacenar la cosecha (Ramsey et al., en prensa).

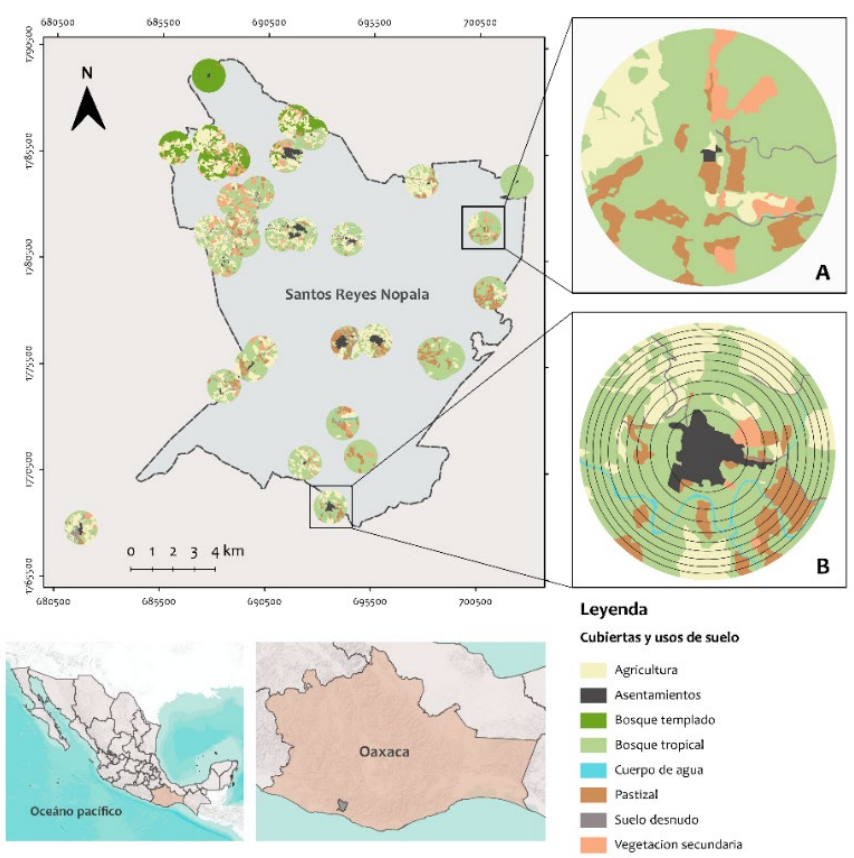

Figura 1. Ubicación de la zona de estudio. Dentro de los círculos se representan las cubiertas y usos de suelo de cada localidad para el año 2004, interpretados en un área de 200 ha (A). Las áreas de influencia para describir la estructura del paisaje se establecieron a partir del centroide de la localidad (B).

Fuente: imágenes satelitales SPOT V, elaboración cartográfica propia. 


\section{Colecta de vectores}

En colaboración con la población, auxiliares y comités de salud comunitarios, el grupo de trabajo implementó un programa de diálogo y concientización sobre la enfermedad de Chagas. Dicho programa incluyó la vigilancia permanente de presencia de vectores en las viviendas y donde mayormente pernoctan los campesinos (parcelas, campo), basado en la capacidad de reconocer (con alto valor predictivo $>95 \%$ ) y colectar las dos especies principales conocidas en el área de estudio (Ramsey et al., 2000). Se solicitó a los pobladores y los comités técnicos comunitarios la colecta de los vectores encontrados dentro de su vivienda o en el hábitat peridoméstico. Los vectores colectados fueron mantenidos en un contenedor de plástico debidamente etiquetado y entregados al personal del grupo de trabajo mediante actores voluntarios de los comités comunitarios.

La infestación doméstica fue estimada utilizando los registros de colecta participativa durante dos periodos de estudio: diciembre de 2003-mayo de 2006 y junio de 2015-febrero de 2016. El índice de infestación que corresponde al porcentaje de viviendas con presencia de al menos un triatomino de cualquier especie entre el total de viviendas revisadas fue calculado para cada una de las localidades (WHO, 2002).

\section{Datos para la caracterización y descripción de la estructura del paisaje}

Se elaboró cartografía de cubiertas y usos del suelo a escala 1:10 000, utilizando como fuente de información imágenes satelitales SPOT V de dos fechas: 25 de abril de 2004 y 24 de febrero de 2015. La temporalidad de las imágenes satelitales corresponde a los periodos de colecta de vectores, por lo que representan la configuración espacial de los elementos del paisaje para cada periodo de estudio.

La cartografía de cubiertas y usos de suelo de la primera fecha, se generó mediante interpretación visual de una superficie circular de 200 ha trazada a partir del centroide de cada localidad como se ilustra en la Figura 1A. La superficie se determinó con base en la revisión de literatura especializada sobre la historia natural de las especies de vectores (Barbu et al., 2010; Ramsey et al., 2000; Ibarra-Cerdeña et al., 2009; Pech-May et al., 2019). La cartografía de la segunda fecha se elaboró siguiendo el método de interpretación interdependiente (Organización de las Naciones Unidas para la Alimentación y la Agricultura -FAO, 2002). Las clases utilizadas para la interpretación fueron las siguientes: asentamientos humanos, pastizales, agricultura, bosque templado, bosque tropical, suelo desnudo y cuerpo de agua. Los asentamientos humanos corresponden al área ocupada con una o más edificaciones utilizadas como viviendas, y en caso de tratarse de un conglomerado, se integraron los elementos naturales y obras materiales como canchas deportivas, escuelas o mercados. Las áreas de pastizales son aquellas donde la vegetación dominante son los pastos, las cuales en su mayoría son de uso ganadero. El bosque de encino y el bosque mixto 
fueron incluidos en la clase de bosque templado, mientras que la selva baja caducifolia y mediana subcaducifolia se catalogaron como bosque tropical. La visualización y manipulación de la información se hizo en el programa ArcGIS 10.1 (ESRI, 2011).

Para la descripción de la estructura del paisaje se calcularon métricas en diez áreas de influencia concéntricas a cada una de las localidades. En la Figura 1B se ejemplifica la delimitación de las áreas de influencia. Se calcularon las siguientes métricas utilizando el programa FRAGSTATS v3 (McGarigal et al., 2012): proporción del paisaje ocupada por cada clase de cubierta o uso de suelo - PLAND - y el grado de fragmentación del bosque tropical — Splitting index, SPLIT—. Se estimó el "efecto del área de influencia" para cada variable estructural mediante una regresión logística, cuya variable dependiente fue la presencia/ausencia del vector en el ambiente doméstico. Dicho efecto alude al área en la cual la estructura del paisaje colindante predice mejor la presencia del vector en el ambiente doméstico (Miguet et al., 2016).

\section{Análisis estadístico}

Se realizaron análisis estadísticos paramétricos y no paramétricos en el programa $\mathrm{R}$ (R Core Team, 2019). Para contrastar la igualdad de proporciones de localidades donde se reportó la colecta de vectores, entre ambos periodos de estudio, se utilizó el estadístico de McNemar. En el caso de los valores del índice de infestación por especie o sin importar la especie de vector, fueron comparados entre ambos periodos de estudio usando la prueba U de Mann-Whitney.

El análisis de la influencia de la estructura del paisaje sobre la presencia de vectores en ambientes domésticos se basó en nueve hipótesis a priori, en las cuales se consideró como variables de análisis la elevación altitudinal de la localidad en msnm, el PLAND de bosque tropical, el PLAND de pastizales, el PLAND de agricultura, la fragmentación del bosque tropical y la densidad de viviendas por ha.

Las hipótesis fueron evaluadas con los siguientes modelos: 1) efecto de la altitud; 2) efecto del PLAND de bosque tropical; 3) efecto del PLAND de pastizales; 4) efecto del PLAND de pastizales y agricultura; 5) efecto de la fragmentación del bosque tropical; 6) efecto de la altitud, la densidad de viviendas y el PLAND de bosque tropical; 7) efecto de la altitud, el PLAND de pastizales y la fragmentación del bosque tropical; 8) modelo global el cual incluye todas las variables sin interacciones; 9) modelo nulo, sin la inclusión de variables explicativas. Para evaluar el soporte de los modelos se usaron modelos lineales generalizados, al igual que el criterio de información de Akaike corregido para tamaños de muestra pequeños, AICc, y los pesos de Akaike, wi. Aquellos modelos con un $\Delta \mathrm{AICc} \leq 6$ entre el mejor modelo y un modelo dado, fueron considerados como el subconjunto de modelos con mayores niveles de soporte empírico. Los parámetros ponderados y sus errores estándar fueron utilizados para calcular la razón de momios —odds ratio_- y sus intervalos de con- 
fianza asociados — 95\% IC — de cada variable de análisis. Esta métrica permite determinar la importancia relativa de cada variable para predecir los datos (Symonds y Moussalli, 2011).

\section{Resultados}

Durante el periodo 2003-2006 el índice de infestación promedio fue de 2,7\% con desviación estándar de 5,6\%, encontrándose vectores en 13 localidades. En el segundo periodo 2015-2016 fueron 15 localidades en las cuales se reportó un índice de infestación promedio de 5,5\% con desviación estándar de 7,7\%. La infestación se mantuvo en diez localidades, mientras que para el periodo 2015-2016 se identificó nueva infestación en cuatro localidades. La variación en el índice de infestación global encontrado entre ambos periodos de estudio no difiere significativamente Mann-Whitney $\mathrm{W}=524, \mathrm{p}>0,05$.

Las dos especies de triatomino colectadas en el ambiente doméstico y sus alrededores en ambos tiempos corresponden a las especies $T$. dimidiata $\mathrm{Hg} 2$ y $T$. mazzottii. En el primer periodo también se colectaron ejemplares aislados de T. bolivari, Panstrongylus rufotuberculatus y Eratyrus cuspidatus. El índice de infestación promedio y la proporción de localidades con presencia del vector $T$. dimidiata no difiere significativamente entre un periodo y otro -McNemar's chi-squared $=0,1666, \mathrm{df}=1, \mathrm{p}>$ 0,05 . Se observó un incremento en el número total de localidades infestadas por esta especie: de 12 localidades con infestación promedio de $2,1 \%$ con desviación estándar de $5,1 \%$, a 14 localidades con una infestación promedio de $2,1 \%$ con desviación estándar de 3,1\% -Mann-Whitney, $\mathrm{W}=560, \mathrm{p}>0,05$. En el caso de T. mazzottii, se reportó un aumento del $100 \%$ en el número de localidades con su presencia en el segundo periodo de estudio, pasando de siete a 14 -McNemar's chi-squared=3,125, $\mathrm{df}=1, \mathrm{p}>0,05$. Durante el periodo 2003-2006 el vector se encontró en siete localidades con un índice de infestación promedio de $0.8 \%$ con desviación estándar de $1,9 \%$, mientras que para el segundo periodo el índice de infestación promedio resultó en un incremento significativo de $4,1 \%$ con desviación estándar de $6,5 \%$, - MannWhitney, $\mathrm{W}=471, \mathrm{p}<0,05$.

Existe un traslape en el rango altitudinal al cual se encuentran ambas especies de vectores dentro del territorio municipal. El límite inferior altitudinal de $T$. dimidiata es de $120 \mathrm{msnm}$ con presencia hasta los $1017 \mathrm{msnm}$ y $1287 \mathrm{msnm}$, en el primero y segundo periodo de estudio, respectivamente. La presencia de la especie $T$. mazzottii en el primer periodo tuvo un rango altitudinal de 216-1017 msnm y en el segundo periodo desde los 120 hasta los $1287 \mathrm{msnm}$. El índice de infestación promedio de la especie T. mazzottii muestra una correlación inversa con la altitud, es decir, los índices de infestación por dicha especie decrecen conforme aumenta la altitud, mientras que para la especie $T$. dimidiata no existe una tendencia clara (Tabla 1). 
Tabla 1

Índice de infestación promedio (SD) de dos especies de vectores de la enfermedad de Chagas de acuerdo con la altitud de la localidad de colecta

\begin{tabular}{crr}
\hline $\begin{array}{c}\text { Rango altitudinal } \\
(\mathrm{msnm})\end{array}$ & Periodo 2003-2006 (SD, N) & Periodo 2015-2016 (SD, N) \\
\hline \multicolumn{3}{c}{ T. dimidiata } \\
\hline $120-556$ & $0,0385(0,0372,9)$ & $0,0580(0,0285,9)$ \\
$557-1017$ & $0,1240(0,1004,3)$ & $0,0361(0,0161,3)$ \\
$1018-1420$ & 0 & $0,0525(0,0063,2)$ \\
\hline $120-556$ & $T$. mazzottii \\
$557-1017$ & $0,0400(0,0243,6)$ & $0,1167(0,0662,8)$ \\
$1018-1420$ & $0,0294(0,1)$ & $0,1052(0,0479,3)$ \\
0 & $0,0910(0,0322,2)$ \\
\hline
\end{tabular}

Fuente: Elaboración propia.

\section{Efecto del área de influencia}

En una primera aproximación el PLAND de áreas agrícolas, pastizales y bosque tropical presentaron bajos valores de AIC y alta devianza explicada en las áreas correspondientes a 180 ha, 200 ha y 20 ha respectivamente (Tabla 2). Es notorio que las variaciones relativas al porcentaje ocupado por bosque tropical son importantes para la infestación en un entorno inmediato al ambiente doméstico. No se encontraron cambios marcados en cuanto a la identificación del "efecto del área de influencia" para el segundo periodo como se compara en la Figura 2. Se detectó un efecto a las 200 ha para el PLAND de áreas agrícolas y pastizales, mientras que el efecto para el PLAND de bosque tropical y fragmentación del mismo fue más evidente en un área de 40 ha.

\section{Tabla 2}

Valores de Devianza y AIC para determinar "efecto de área de influencia" de las variables de la estructura del paisaje

\begin{tabular}{ccc}
\hline $\begin{array}{c}\text { Variable de la estructura del } \\
\text { paisaje }\end{array}$ & Periodo 2003-2006 & Periodo 2015-2016 \\
\hline Porcentaje de agricultura & $6,33(47,25)$ & $8,85(47,56)$ \\
Porcentaje de pastizal & $3,57(48,52)$ & $0,22(51,69)$ \\
Porcentaje de bosque tropical & $1,13(49,65)$ & $3,87(49,95)$ \\
Fragmentación del bosque & $19,59(41,12)$ & $10,61(45,71)$ \\
\hline
\end{tabular}

Fuente: Elaboración propia. 

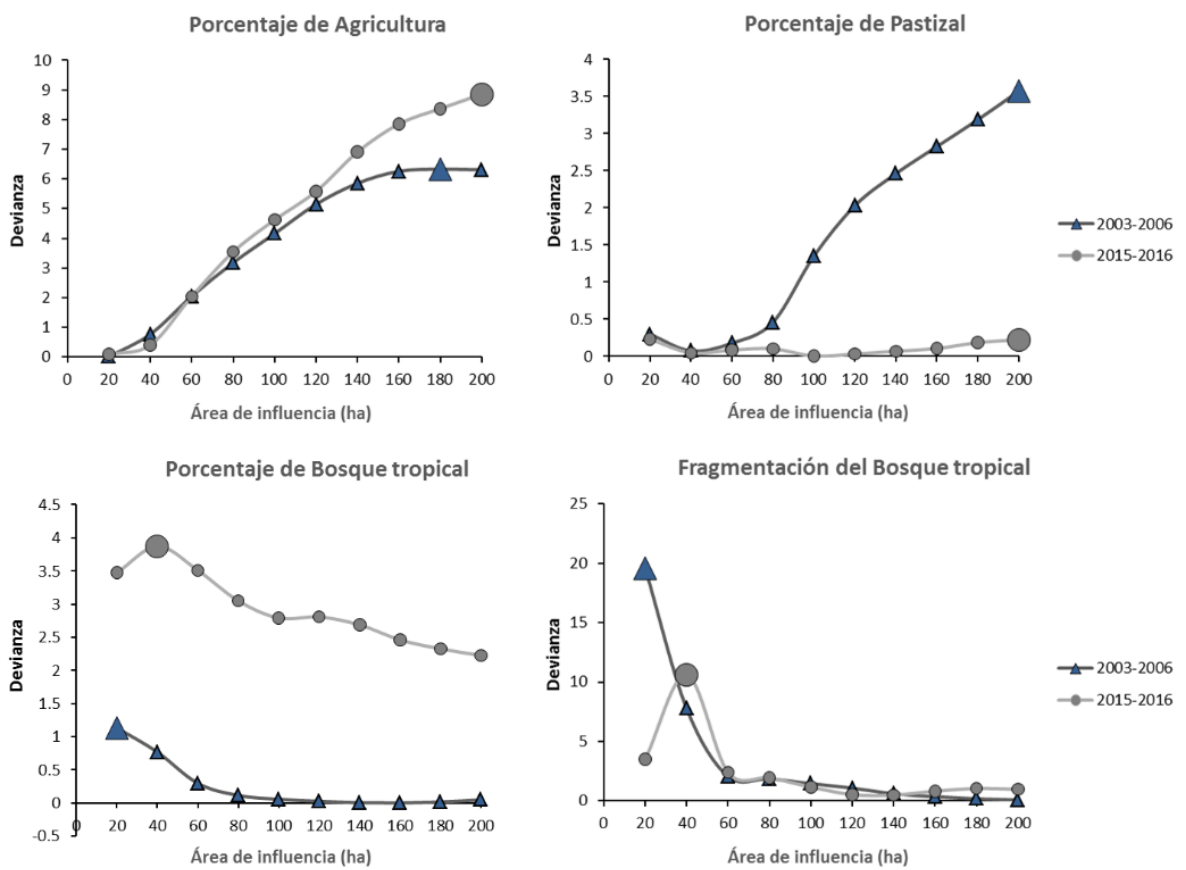

Figura 2. Efecto del área de influencia en la cual la estructura del paisaje predice mejor la presencia de vectores en el ambiente doméstico. El símbolo de mayor tamaño indica el área de influencia con el valor de devianza más grande y el valor de AIC más bajo.

Fuente: elaboración propia.

\section{Factores que influyen en la infestación de las localidades}

La identificación de los modelos a priori que mejor predicen la presencia del vector en ambientes domésticos se realizó en dos etapas, de acuerdo con el período de estudio. En ninguna etapa hubo multicolinearidad entre las variables, $\mathrm{VIF}<4$. Los modelos 8,6 y 7 fueron los que presentaron mayor soporte para el primer periodo, $\Delta$ AICc $\leq 6$. Estos modelos explican el 77,8\%, 52,7\% y el $48 \%$ de la variación en la probabilidad de presencia de un vector en una localidad, respectivamente (Tabla 3). En esta primera etapa de selección de modelos, la densidad de viviendas y la altitud a la cual se encuentra la localidad son variables con un efecto importante en la presencia del vector en las localidades (Tabla 4). Se identificó una asociación de protección estadísticamente significativa, lo que sugiere que la presencia del vector en ambientes domésticos de las localidades de estudio disminuye cuando la altitud y la densidad de viviendas aumentan. En las localidades donde la elevación altitudinal es mayor a los $1122 \mathrm{msnm}$ y existían más de 21 viviendas/ha no hubo infestación doméstica. 
Tabla 3

Modelos a priori que explican la presencia del vector en el ambiente doméstico durante el periodo de estudio 2003-2006

\begin{tabular}{|c|c|c|c|c|c|c|}
\hline Modelo & Variable & $K$ & $\log _{e}(L)$ & $A I C_{c}$ & $\Delta A I C_{c}$ & $w_{i}$ \\
\hline Global * & Todas las variables & 7 & $-6,0501$ & 26,2718 & 0,0000 & 0,8077 \\
\hline $6 *$ & $\begin{array}{l}\text { Efecto de la altitud, la } \\
\text { densidad de viviendas y el } \\
\text { porcentaje de bosque tropical } \\
\text { en } 20 \text { ha }\end{array}$ & 4 & $-10,9200$ & 29,9009 & 3,6292 & 0,1316 \\
\hline $7 *$ & $\begin{array}{l}\text { Efecto de la altitud, el } \\
\text { porcentaje de pastizal en } 200 \\
\text { ha y la fragmentación del } \\
\text { bosque tropical en } 20 \text { ha }\end{array}$ & 4 & $-11,9203$ & 31,9015 & 5,6298 & 0,0484 \\
\hline 1 & Efecto de la altitud & 2 & $-15,4093$ & 34,8368 & 8,5650 & 0,0112 \\
\hline 5 & $\begin{array}{l}\text { Fragmentación del bosque } \\
\text { tropical en } 20 \text { ha }\end{array}$ & 2 & $-18,5646$ & 41,1474 & 14,8757 & 0,0005 \\
\hline 4 & $\begin{array}{l}\text { Efecto del porcentaje de } \\
\text { pastizal en } 200 \text { ha y el } \\
\text { porcentaje de agricultura en } \\
180 \text { ha }\end{array}$ & 3 & $-18,5044$ & 43,0454 & 16,7736 & 0,0006 \\
\hline Nulo & & 1 & $-23,0899$ & 48,1859 & 21,9141 & 0,0001 \\
\hline 3 & $\begin{array}{l}\text { Efecto del porcentaje de } \\
\text { pastizal en } 200 \text { ha }\end{array}$ & 2 & $-22,2645$ & 48,5473 & 22,2755 & 0,0000 \\
\hline 2 & $\begin{array}{l}\text { Efecto del porcentaje de } \\
\text { bosque tropical en } 20 \text { ha }\end{array}$ & 2 & $-22,8284$ & 49,6750 & 23,4032 & 0,0000 \\
\hline
\end{tabular}

$K$, número de parámetros estimado para cada modelo; $\log _{e}(L)$, función logística de máxima verosimilitud; $A I C_{c}$, criterio de información de Akaike; $\triangle A I C_{c}$, diferencia de $\mathrm{AIC}_{\mathrm{c}} \mathrm{y} w_{i}$ pesos de Akaike. Con el símbolo * se indican los modelos de mayor soporte.

Fuente: Elaboración propia.

Fueron cinco los modelos equivalentes al mejor modelo con $\triangle \mathrm{AICc} \leq 2$ de la selección de modelos de la segunda etapa de evaluación (Tabla 5). El modelo con mayor soporte fue el que incluye el efecto de la fragmentación del bosque tropical a 40 ha, el modelo 5. Sin embargo, todos los modelos, incluyendo el modelo global y nulo, tienen un $\triangle \mathrm{AICc} \leq 6$. La variabilidad explicada por el mejor modelo es del $11,17 \%$, aunque el modelo global explica el 23,33\% de la variación en la probabilidad de presencia del vector en el ambiente doméstico de las localidades (Tabla 5). Se sugieren como variables importantes la altitud y el porcentaje ocupado por el bosque tropical en un entorno inmediato al ambiente doméstico, debido a que los intervalos de confianza de la razón de momios no tienen un traslape con uno o el traslape es ligero (Tabla 6). En este caso, la asociación de protección sugiere que la presencia del vector en el ambiente doméstico disminuye conforme el porcentaje ocupado por el bosque tropical aumenta. 
Tabla 4

Coeficientes de regresión de los modelos promediados (COEF), error estándar (SE), razón de momios (OR) e intervalos de confianza (IC) del $95 \%$, a partir de los modelos a priori durante el periodo 2003-2006

\begin{tabular}{lcccc}
\hline \multicolumn{1}{c}{ Variable } & $\Sigma w_{i}$ & COEF & EE & OR (IC 95 \%) \\
\hline Altitud & 0,9988 & $-0,0055$ & 0,0034 & $0,9945(0,9879-1,0011) *$ \\
Porcentaje de Bosque tropical en 20 ha & 0,9393 & 0,0572 & 0,1014 & $1,0588(0,8680-1,2917)$ \\
Porcentaje de Pastizal en 200 ha & 0,8567 & 0,2359 & 0,1693 & $1,2660(0,9086-1,7641)$ \\
Porcentaje de Agricultura en 180 ha & 0,8083 & 0,3230 & 0,1937 & $1,3813(0,9451-2,0190)$ \\
Fragmentación del bosque tropical & 0,8566 & 0,0201 & 0,0326 & $1,0203(0,9571-1,0876)$ \\
en 20 ha & 0,9393 & $-0,3386$ & 0,1982 & $0,7128(0,4833-1,0513) *$ \\
Densidad de viviendas & & & &
\end{tabular}

Con el símbolo * se indican las variables consideradas como importantes.

Fuente: Elaboración propia.

Tabla 5

Modelos a priori que explican la presencia del vector en el ambiente doméstico durante el periodo de estudio 2015-2016

\begin{tabular}{|c|c|c|c|c|c|c|}
\hline Modelo & Variable & $K$ & $\log _{e}(L)$ & $A I C_{c}$ & $\triangle A I C_{c}$ & $w_{i}$ \\
\hline $5 *$ & $\begin{array}{l}\text { Fragmentación del bosque tropical } \\
\text { en } 40 \text { ha }\end{array}$ & 2 & $-21,2317$ & 46,4816 & 0,0000 & 0,1598 \\
\hline $1 *$ & Efecto de la altitud & 2 & $-21,3266$ & 46,6714 & 0,1898 & 0,1453 \\
\hline $6 *$ & $\begin{array}{l}\text { Efecto de la altitud, la densidad de } \\
\text { viviendas y el porcentaje de bosque } \\
\text { tropical en } 40 \text { ha }\end{array}$ & 4 & $-19,3715$ & 46,8040 & 0,3225 & 0,1360 \\
\hline $7 *$ & $\begin{array}{l}\text { Efecto de la altitud, el porcentaje de } \\
\text { pastizal en } 200 \text { ha y la fragmentación } \\
\text { del bosque tropical en } 40 \text { ha }\end{array}$ & 4 & $-19,4704$ & 47,0018 & 0,5202 & 0,1232 \\
\hline $4 *$ & $\begin{array}{l}\text { Efecto del porcentaje de pastizal en } \\
200 \text { ha y el porcentaje de agricultura } \\
\text { en } 200 \text { ha }\end{array}$ & 3 & $-20,5870$ & 47,2105 & 0,7289 & 0,1110 \\
\hline Nulo & & 1 & $-23,9018$ & 49,8096 & 3,3281 & 0,0908 \\
\hline 2 & $\begin{array}{l}\text { Efecto del porcentaje de bosque } \\
\text { tropical en } 40 \text { ha }\end{array}$ & 2 & $-22,9762$ & 49,9707 & 3,4891 & 0,1675 \\
\hline Global & Todas las variables & 7 & $-18,3257$ & 50,8229 & 4,3413 & 0,0547 \\
\hline 3 & $\begin{array}{l}\text { Efecto del porcentaje de pastizal en } \\
200 \text { ha }\end{array}$ & 2 & $-23,8486$ & 51,7152 & 5,2337 & 0,0117 \\
\hline
\end{tabular}

$K$, número de parámetros estimado para cada modelo; $\log _{e}(L)$, función logística de máxima verosimilitud; $A I C_{c}$, criterio de información de Akaike; $\triangle A I C_{c}$, diferencia de $\mathrm{AIC}_{\mathrm{c}} \mathrm{y} w_{i}$ pesos de Akaike. Con el símbolo * se indican los modelos de mayor soporte.

Fuente: Elaboración propia. 
Tabla 6

Coeficientes de regresión de los modelos promediados (COEF), errores estándar (SE), razón de momios (OR) e intervalos de confianza (IC) del 95\%, a partir de los modelos $a$ priori durante el periodo 2015-2016

\begin{tabular}{lcccc}
\hline \multicolumn{1}{c}{ Variable } & $\Sigma w_{i}$ & COEF & $E E$ & OR (IC 95\%) \\
\hline Altitud & 0,4592 & $-0,0024$ & 0,0012 & $0,9976(0,9952-1,0000)^{*}$ \\
$\begin{array}{l}\text { Porcentaje de Bosque tropical } \\
\text { en 40 ha }\end{array}$ & 0,3582 & $-0,0244$ & 0,0193 & $0,9759(0,9397-1,0136)^{*}$ \\
$\begin{array}{l}\text { Porcentaje de Pastizal en 200 } \\
\text { ha }\end{array}$ & 0,3006 & 0,0294 & 0,0538 & $1,0299(0,9268-1,1444)$ \\
$\begin{array}{l}\text { Porcentaje de Agricultura en } \\
\text { 200 ha }\end{array}$ & 0,1657 & 0,0597 & 0,0375 & $1,0615(0,9863-1,1425)$ \\
$\begin{array}{l}\text { Fragmentación del bosque } \\
\text { tropical en 40 ha }\end{array}$ & 0,3377 & 0,0062 & 0,0039 & $1,0062(0,9984-1,0140)$ \\
$\begin{array}{l}\text { Densidad de viviendas por ha } \\
\text { pendidien }\end{array}$ & 0,1907 & 0,0238 & 0,0805 & $1,0241(0,8746-1,1990)$ \\
\hline
\end{tabular}

Con el símbolo * se indican las variables consideradas como importantes.

Fuente: Elaboración propia.

\section{Discusión y conclusiones}

El riesgo de contacto vector-humano en ambientes domésticos no es enteramente explicado por la calidad y carencias de la vivienda (Enger et al., 2004; Ramsey et al., 2005; Cohen et al., 2006; Bustamante et al., 2009; Dumonteil et al., 2013; Grijalva et al., 2017), como la literatura temprana sobre la epidemiologia de la enfermedad de Chagas en Sudamérica lo señalaba. En la actualidad varios estudios señalan que una proporción de la transmisión de T. cruzi al humano no ocurre en el hábitat doméstico, sino en áreas de ecotono o silvestres (Ramsey et al., 2012; López-Cancino et al., 2015; Valdez-Tah et al., 2015a, b). Los resultados del presente estudio muestran evidencia de que la pérdida de bosque tropical y sus variaciones espaciales en un entorno inmediato al ambiente doméstico, en combinación con una baja densidad de vivienda, y la elevación altitudinal, están asociados con un incremento en la presencia doméstica de las dos especies más abundantes de triatominos.

Históricamente en el municipio de Santos Reyes Nopala se ha reportado la presencia de tres especies de vectores (Ramsey et al., 2000; Ibarra-Cerdeña et al., 2009). Una era invasora, Rhodnius prolixus (Hashimoto y Schofield, 2012) y no se ha colectado en México desde 2005, mientras T. dimidiata y T. mazzottii han sido las dos especies con una dispersión y capacidad sinantrópica más importantes. En los 10 años que hubo entre las evaluaciones del presente estudio se ha presentado una ampliación en el gradiente altitudinal de la distribución de ambas especies de vectores, llegando hasta los $1785 \mathrm{msnm}$ para $T$. dimidiata y $1395 \mathrm{msnm}$ para $T$. mazzottii. La evidencia 
encontrada en este estudio indica que la infestación a nivel municipal se ha duplicado con el tiempo y que dicho cambio se debe principalmente a un incremento en la infestación por el vector T. mazzottii en altitudes menores. Se trata de una especie distribuida tanto en bosques tropicales como en templados y con alta capacidad para alimentarse de sangre de aves con un desarrollo ventajoso (Ibarra-Cerdeña et al., 2009). Con un incremento del $100 \%$ en el número de localidades infestadas por $T$. mazzottii, al igual que un aumento significativo en el porcentaje de viviendas infestadas — de $0,8 \%$ a $4,1 \%$ - esta especie es el vector predominante no sólo en el municipio, sino también en el estado de Oaxaca (Ramsey et al., 2015).

La deforestación de los bosques tropicales para su conversión en zonas agropecuarias favorece la presencia de vectores en el ambiente doméstico (Ibarra-Cerdeña et al., 2017) como se ha reportado en paisajes rurales de Panamá (Gottenker et al., 2011) y Argentina (Vázquez-Prokopec et al., 2012). Los bosques tropicales albergan una gran variedad de hospederos reservorios del parásito T. cruzi y hospederos de los vectores, mamíferos silvestres (Izeta-Alberdi et al., 2016). Los diferentes procesos de cambio de esta cubierta modifican las comunidades de hospederos en términos de abundancia y diversidad (Ibarra-Cerdeña et al., 2017). Los animales pecuarios y mascotas (ganado, gallinas, perros, gatos) y la misma población humana son alimento abundante y continuo para los vectores, es por ello que las actividades de pastoreo y la explotación de los recursos forestales, como la recolección de leña y cacería, han sido documentadas como factores asociados a elevados índices de infestación doméstica (Vázquez-Prokopec et al., 2012). Aunque en el presente estudio las zonas agrícolas o los pastizales per se no tuvieron efecto significativo en la infestación de las localidades, se ha hipotetizado que estas actividades podrían desempeñar un papel importante en la incidencia de la enfermedad en algunos estados del sur de México (Ramsey et al., 2012; López-Cancino et al., 2015). La agricultura es una de las principales actividades ocupacionales de la población y es en estas áreas de trabajo donde se sugiere que puede tener lugar la transmisión vectorial (Ibañez-Cervantes et al., 2018).

Los factores socioeconómicos no fueron incluidos en este trabajo debido a que la mayoría de las viviendas en el municipio cuentan con las características de áreas, documentadas en estudios previos, que presentan altos porcentajes de infestación (Ramsey et al., 2002; Bustamante et al., 2014). Los esfuerzos gubernamentales para abatir la pobreza en el municipio se reflejan en el mejoramiento de las condiciones de las viviendas y el suministro de servicios básicos, debido a la implementación de diversos programas para dignificar dicho espacio (Ayuntamiento de Nopala, 2014). Sin embargo, estos programas han promovido la construcción de viviendas - frecuentemente sin la destrucción de viviendas viejas - y el subsecuente desarrollo de las actividades productivas, lo que puede tener consecuencias directas o indirectas (positivas o negativas) sobre la prevalencia de infestación. Un ejemplo de ello es el 
caso particular de la localidad El Aguacatal, ubicada al noroeste de la cabecera municipal. En esta localidad se registró la colecta del vector $T$. dimidiata únicamente para el periodo 2015-2016, con una infestación doméstica de 5,8\%. La deforestación fue un proceso predominante alrededor de la localidad desde 2011 debido a la expansión de la frontera agrícola como se ilustra en la Figura 3 (SAGARPA y FAO, 2015).

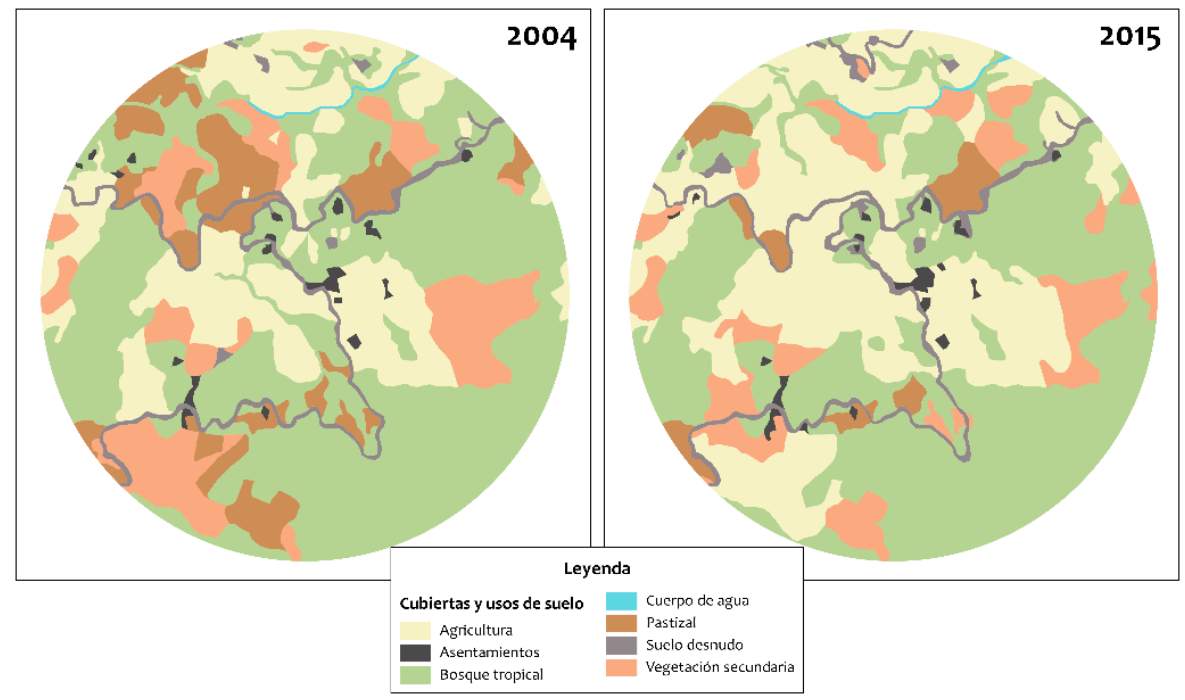

Figura 3. Comparativa del paisaje en El Aguacatal, Santos Reyes Nopala, donde se muestra la expansión de las áreas agrícolas (cultivo de caña de azúcar).

Fuente: imágenes satelitales SPOT V, elaboración cartográfica propia.

En México, desde el 2007 se ha documentado un aumento considerable en la detección de nuevos casos de la enfermedad de Chagas, principalmente debido al incremento en investigación y presencia de grupos académicos en las comunidades, y en menor medida a programas estatales encaminados a la detección de la presencia del vector en vivienda y a actividades para eliminar la transmisión transfusional (IbañezCervantes et al., 2018). Actualmente, las estrategias y líneas de acción para el control de la transmisión intradomiciliar se basan en la estratificación de riesgo (a pesar de no tener definición clara de esta), mejoramiento de la vivienda y control químico del vector en áreas prioritarias, definidas así por ser endémicas y por tener la necesidad de acciones de respuesta (CENAPRECE, 2018). Los resultados que aquí se presentan pueden ayudar a enriquecer la definición de riesgo de exposición al vector en México, de manera que se sugiere priorizar las estrategias de información y de vigilancia co- 
munitaria de presencia y contacto en las localidades de menor altitud de este municipio. Es en estos lugares donde prevalece el movimiento continuo entre el ambiente doméstico y los cultivos o el bosque, y una carencia por calidad y servicios básicos de la vivienda, más aún en los asentamientos pequeños (Ramsey, en prensa). Acciones como el almacenaje de alimentos y confinamiento de animales en lugares lejanos del ambiente doméstico, al igual que el uso de pabellones durante la noche en el caso de dormir en las áreas de pastoreo, ayudarán a prevenir el contacto de los humanos con el vector. Por lo tanto es importante tomar medidas preventivas en el paisaje completo debido a las diferentes relaciones que los pobladores establecen con su entorno natural (Valdez-Tah et al., 2015b).

El control de poblaciones de vectores requiere una apropiada estrategia de implementación, cobertura, aceptación y uso de medidas de control para generar un impacto adecuado. En el presente trabajo se enfatiza la comprensión de las características del paisaje que propician la presencia en el hábitat doméstico y el peligro de contacto entre el vector y el humano en un contexto local. El enfoque de paisaje con la finalidad de determinar patrones espaciales relacionados con la amplificación de riesgo de enfermedades trasmitidas por vectores ha sido poco abordado e incluido en estudios epidemiológicos o eco-epidemiológicos. El uso de dicho enfoque brinda una oportunidad para discriminar los diferentes componentes del paisaje que influyen en la infestación doméstica y provee evidencia para dar a conocer a la población y con ello cambiar prácticas peligrosas. Se debe fomentar el diálogo entre la población y los diferentes sectores institucionales para evitar prácticas y programas que promuevan o amplifiquen la infestación (agropecuario, de salud y académico). Es a través de los esfuerzos intersectoriales e interinstitucionales que se fortalecen los programas y actividades de gobierno que mitigan la exposición, aunque las prácticas y cambios estructurales o de vigilancia permitirán reducir la vulnerabilidad para la transmisión vectorial en procesos locales.

\section{Bibliografía}

Ayuntamiento de Nopala (2014). Plan municipal de desarrollo 2014-2016, Santos Reyes Nopala, Oaxaca.

Barbu C., Dumonteil E. \& Gourbière S. (2010). "Characterization of the dispersal of non-domiciliated Triatoma dimidiata through the selection of spatially explicit models", PLoS Neglected Tropical Diseases, 4(8), 777. https://doi.org/10.1371/journal.pntd.0000777

Brito R.N., Gorla D.E., Diotaiuti L., Gomes A.C.F., Souza R.C.M. \& Abad-Franch F. (2017). "Drivers of house invasion by sylvatic Chagas disease vectors in the Amazon-Cerrado transition: A multi-year, state-wide assessment of municipalityaggregated surveillance data", PLoS Neglected Tropical Diseases, 11(11), e0006035. https://doi.org/10.1371/journal. pntd.0006035 
Bustamante D.M., De Urioste-Stone S.M., Juárez J.G. \& Pennington P.M. (2014). "Ecological, social and biological risk factors for continued Trypanosoma cruzi transmission by Triatoma dimidiata in Guatemala", PLoS ONE, 9(8), e104599. https://doi.org/10.1371/journal.pone.0104599

Castro F., Bunting S.C., Strand E. \& Godinho-Ferreira P. (eds.) (2019). Applied Landscape Ecology (1a), UK. Editorial Wiley, pp. 461.

CENAPRECE (2018). "Centro Nacional de Programas Preventivos y Control de Enfermedades. Avances, logros y retos 2013-2018”, Secretaría de Salud, México. Recuperado de https://www.gob.mx/cms/uploads/attachment/file/398612/ALR CENAPRECE opt 1.pdf

Cohen J.M., Wilson M.L., Cruz-Celis A., Ordoñez R. \& Ramsey J. M. (2006). “Infestation by Triatoma pallidipennis (Hemiptera: Reduviidae: Triatominae) is associated with housing characteristics in rural Mexico", Journal of Medical Entomology, 43(6): 1252-1260. https://doi.org/10.1093/jmedent/43.6.1252

CONAPO (2010). "Índice de marginación por entidad federativa y municipio 2010".

Recuperado de http://www.cnapo.gob.mx/en/CONAPO/Indices_de_Marginacion_2010_por_entidad_federativa_y_municipio

Dumonteil E., Nouvellet P., Rosecrans K., Ramirez-Sierra M.J., Gamboa-León R., Cruz-Chan V., Rosado-Vallado M. \& Gourbière, S. (2013). "Eco-Bio-Social determinants for house infestation by non-domiciliated Triatoma dimidiata in the Yucatan Peninsula, Mexico", PLoS Neglected Tropical Diseases, 7(9), e2466. https://doi.org/10.1371/journal.pntd.0002466

Dumonteil E., Ramírez-Sierra M.J., Ferral J., Euan-Garcia M. \& Chavez-Nuñez L., (2009). Usefulness of community participation for the fine temporal monitoring of house infestation by non-domiciliated triatomines. The Journal of Parasitology, 95(2), 469-471. https://doi.org/10.1645/GE-1712.1

Enger K.S., Ordoñez R., Wilson M.L. \& Ramsey J.M. (2004). "Evaluation of risk factors for rural infestation by Triatoma pallidipennis (Hemiptera: Triatominae), a Mexican vector of Chagas disease", Journal of Medical Entomology, 41: 760767. https://doi.org/10.1603/0022-2585-41.4.760

ESRI (2011). ArcGIS Desktop: Release 10. Redlands, CA: Environmental Systems Research Institute.

FAO (2002). "FRA2000, pan-tropical survey of forest cover changes 1980-2000 methods and implementation, FAO FRA working paper 49a”, Rome. Recuperado de http://www.fao.org/3/a-ak592e.pdf

Gottdenker N.L., Calzada J.E., Saldaña A. \& Carroll C.R. (2011). “Association of anthropogenic land use change and increased abundance of the Chagas disease vector Rhodnius pallescens in a rural landscape of Panama", American Journal of Tropical Medicine and Hygiene, 84(1): 70-77. https://doi.org/10.4269/ajtmh.2011.10-0041. 
Grijalva M.J., Villacıs A.G., Moncayo A.L., Ocaña-Mayorga S., Yumiseva C.A., Baus E.G. (2017). "Distribution of triatomine species in domestic and peridomestic environments in central coastal Ecuador", PLoS Negl Trop Dis, 11(10): e0005970.

Hashimoto K. \& Schofield C.J. (2012). "Elimination of Rhodnius prolixus in Central America", Parasites \& Vectors, 5: 45. https://doi.org/10.1186/1756-3305-5-45

Ibarra-Cerdeña C.N., Sánchez-Cordero V., Peterson A. T. \& Ramsey, J.M., (2009). "Ecology of North American Triatominae", Acta Tropica, 110: 178-186. https://doi.org/10.1016/j.actatropica.2008.11.012

Ibarra-Cerdeña C.N., Valiente-Banuet L., Sánchez-Cordero V., Stephens C.R. \& Ramsey J.M. (2017). “Trypanosoma cruzi reservoir-triatomine vector co-occurrence networks reveal meta-community effects by synanthropic mammals on geographic dispersal", PeerJ, 5, e3152. https://doi.org/10.7717/peerj.3152

INEGI (2009). "Prontuario de información geográfica municipal de los Estados Unidos Mexicanos. Santos Reyes Nopala, Oaxaca”. Recuperado de http://www3.inegi.org.mx/contenidos/app/mexicocifras/datos_geograficos/20/20526.pdf

(2010). Censo de Población y Vivienda 2010. Principales resultados por localidad (ITER). Recuperado de http://www.inegi.org.mx/sistemas/consulta resultados/iter2010.aspx

Izeta-Alberdi I., Ibarra-Cerdeña C.N., Moo-Llanes D.M. \& Ramsey J.M. (2016). "Geographical, landscape and host associations of Trypanosoma cruzi DTUs and lineages", Parasites \& Vectors, 9: 631.

https://doi.org/10.1186/s13071-016-1918-2

López-Cancino S.A., Tun-Ku E., De la Cruz-Felix H.K., Ibarra-Cerdeña C.N., IzetaAlberdi A., Pech-May A., Mazariegos-Hidalgo C.J., Valdez-Tah A. \& Ramsey J. M. (2015). "Landscape ecology of Trypanosoma cruzi in the southern Yucatan Peninsula", Acta Tropica, 151: 58-72.

https://doi.org/10.1016/j.actatropica.2015.07.021

McGarigal K., Cushman S.A. \& Ene E., (2012). FRAGSTATS v4: Spatial pattern analysis program for categorical maps and continuous maps, Amherst, University of Massachusetts.

Miguet P., Jackson H.B., Jackson N.D., Martin A.E. \& Fahrig L. (2016). "What determines the spatial extent of landscape effects on species?", Landscape Ecology, 31: 1177-1194. https://doi.org/10.1007/s10980-015-0314-1

Pech-May A., Mazariegos-Hidalgo C.J., Izeta-Alberdi A., López-Cancino S.A., TunKu E., De la Cruz-Felix K., Ibarra-Cerdeña C.N., González R.E. \& Ramsey J.M., (2019). "Genetic variation and phylogeography of the Triatoma dimidiata complex evidence a potential center of origin and recent divergence of haplogroups 
having differential Trypanosoma cruzi and DTU infections", PLoS Neglected Tropical Diseases, 13(1). https://doi.org/10.1371/journal.pntd.0007044

Pérez J.G. (ed.) (2010). Buscando el origen de los Chatinos de Nopala (3a), Oaxaca, México, Editorial Carteles, pp. 160.

Ramírez-Sierra M.J., Herrera-Aguilar M., Gourbière S. \& Dumonteil E. (2010). "Patterns of house infestation dynamics by non-domiciliated Triatoma dimidiata reveal a spatial gradient of infestation in rural villages and potential insect manipulation by Trypanosoma cruzi", Tropical Medicine and International Health, 15(1): 77-86. https://doi.org/10.1111/j.1365-3156.2009.02422.x

R Core Team, (2019). R: A language and environment for statistical computing. $\mathrm{R}$ Foundation for Statistical Computing, Vienna, Austria.

Ramsey J.M., Ordoñez R., Cruz-Celis A., Alvear A. L., Chavez V., Lopez R., Pintor J. R., Gama F. \& Carrillo S. (2000). "Distribution of domestic Triatominae and stratification of Chagas Disease transmission in Oaxaca, Mexico", Medical and Veterinary Entomology, 14: 19-30.

https://doi.org/10.1046/j.1365-2915.2000.00214.x

Ramsey J.M., Alvear A.L., Ordoñez R., Muñoz G., Garcia A., Lopez R. \& Leyva R., (2005). "Risk factors associated with house infestation by the Chagas disease vector Triatoma pallidipennis in Cuernavaca metropolitan area, Mexico", Medical and Veterinary Entomology, 19: 219-228.

https://doi.org/10.1111/j.0269-283X.2005.00563.x

Ramsey J.M., Gutierrez-Cabrera A.E., Salgado-Ramírez L., Peterson A.T., SanchezCordero V. \& Ibarra-Cerdeña C.N. (2012). "Ecological connectivity of Trypanosoma cruzi reservoirs and Triatoma pallidipennis hosts in an anthropogenic landscape with endemic Chagas Disease", PLoS ONE, 7(9): e46013. https://doi.org/10.1371/0046013

Ramsey J.M., Peterson A.T., Carmona-Castro O., Moo-Llanes D.A., Nakazawa Y., Butrick M., Tun-Ku E., De la Cruz-Félix K. \& Ibarra-Cerdeña C.N. (2015). “Atlas of Mexican Triatominae (Reduviidae: Hemiptera) and vector transmission of Chagas disease", Memórias Do Instituto Oswaldo Cruz, 110(3): 339-352. https://doi.org/10.1590/0074-02760140404

Rossi J.C., Duarte E.C. \& Gurgel-Gonçalves R. (2015). "Factors associated with the occurrence of Triatoma sordida (Hemiptera: Reduviidae) in rural localities of Central-West Brazil", Memórias Do Instituto Oswaldo Cruz, 110(2): 192-200. https://doi.org/10.1590/0074-02760140395

SAGARPA \& FAO (2015). PESA en México. Recuperado de www.pesamexico.org/PESAenMéxico/ElPESAenMéxicoysumetodología.aspx

Sánchez-González G., Figueroa-Lara A., Elizondo-Cano M., Wilson L., NoveloGarza B., Valiente-Banuet L. \& Ramsey J.M., (2016). "Cost-Effectiveness of Blood Donation Screening for Trypanosoma cruzi in Mexico", PLoS Neglected 
Tropical Diseases, 10(3): e0004528.

https://doi.org/10.1371/journal.pntd.0004528

Schmunis G. \& Yadon Z.E. (2010). "Chagas disease: a Latin American health problem becoming a world health problem”, Acta Tropica, 115(1-2): 14-21. https://doi.org/10.1016/j.actatropica.2009.11.003

Symonds M.R.E. \& Moussalli A. (2011). "A brief guide to model selection, multimodel inference and model averaging in behavioural ecology using Akaike's information criterion", Behavioral Ecology and Sociobiology, 65: 13-21. https://doi.org/10.1007/s00265-010-1037-6

Valdez-Tah A., Huicochea-Gómez L., Nazar-Beutelspacher A., Ortega-Canto J. \& Ramsey J.M. (2015a). "La vulnerabilidad humana a la transmisión vectorial de Trypanosoma cruzi a través de los procesos de salud-enfermedad y la apropiación social del territorio", Salud Colectiva, 11(2): 191-210. https://doi.org/10.18294/sc.2015.683

Valdez-Tah A., Huicochea-Gómez L., Ortega-Canto J., Nazar-Beutelspacher A. \& Ramsey J.M. (2015b). "Social representations and practices towards Triatomines and Chagas Disease in Calakmul, México", PLoS ONE, 10(7): e0132830. https://doi.org/10.1371/journal.pone.0132830

Vázquez-Prokopec G.M., Spillmann C., Zaidenberg M., Gürtler R.E. \& Kitron U., (2012). "Spatial heterogeneity and risk maps of community infestation by Triatoma infestans in rural northwestern Argentina”, PLoS Neglected Tropical Diseases, 6(8): e1788. https://doi.org/10.1371/journal.pntd.0001788

WHO (2002). "Control of Chagas Disease. Technical Report Series 905”. Second report of the WHO Expert Committee. Ginebra. World Health Organization, pp. 120. Recuperado de https://apps.who.int/iris/bitstream/handle/10665/42443/WHO_TRS_905.pdf? sequence=1

WHO (2015). "Chagas disease (American trypanosomiasis)". Recuperado de http://www.who.int/mediacentre/factsheets/fs340/en/

WHO (2017). "Respuesta mundial para el control de vectores 2017-2030". Recuperado de https://www.who.int/malaria/areas/vector_control/Draft-WHO-GVCR2017-2030-esp.pdf 
